\title{
Exportaciones manufactureras mexicanas por nivel tecnológico y su efecto sobre el empleo en 2008 y 2012: un análisis de descomposición estructural
}

\author{
Recibido: marzo, 05 de 2018 - Aprobado: mayo, 30 de 2018
}

DoI: http://dx.doi.org/10.12804/revistas.urosario.edu.co/economia/a.7206

\author{
Brenda Murillo-Villanueva* \\ Martín Puchet-Anyul ${ }^{+}$ \\ Gerardo Fujii-Gamberoł
}

\section{Resumen}

La composición de las exportaciones manufactureras por nivel tecnológico se usó para estimar, a través del análisis de descomposición estructural y del valor de la producción bruta de México de 2008 y 2012, los cambios en el empleo por unidad de variación en las exportaciones de cada subsector. Los hallazgos fundamentales son tres: primero, que son los subsectores manufactureros de media y alta tecnología los que generan menos empleos por unidad de exportación; segundo, que son estos subsectores los que incrementaron en mayor medida sus exportaciones de forma que sus requerimientos de empleo aumentaron más que los del resto de los subsectores; y tercero, que el cambio observado en las exportaciones ha tenido un efecto neto positivo en la creación de empleo.

Palabras clave: exportaciones manufactureras, niveles tecnológicos, empleo, análisis de descomposición estructural.

Clasificación JEL: F14, F16.

* Universidad Nacional Autónoma de México. Correo electrónico: bmv_17_5@ hotmail.com

† Universidad Nacional Autónoma de México. Correo electrónico: anyul@unam.mx

‡ Universidad Nacional Autónoma de México. Correo electrónico: fujii@unam.mx

Cómo citar este artículo: Murillo-Villanueva, B., Puchet-Anyul, M., \& Fujii-Gambero, G. (2018). Exportaciones manufactureras mexicanas por nivel tecnológico y su efecto sobre el empleo en 2008 y 2012: un análisis de descomposición estructural. Revista de Economía del Rosario, 21(2), 409-433.

DoI: http://dx.doi.org/10.12804/revistas.urosario.edu.co/economia/a.7206 


\title{
Manufacturing Exports by Technological Level and Employment in Mexico between 2008 and 2012: An Analysis of Structural Decomposition
}

\begin{abstract}
The composition of the manufacturing exports by technological level was utilized to estimate, by means of the structural decomposition analysis and the gross production in México of 2008 and 2012, the changes on employment per unit of variation in each subsector's exports. The main findings are three: first, that the manufacturing subsectors of medium and high technology generate the less number of jobs per unit of export; second, that the medium and high technology subsectors increased the most their exports, leading to higher requirements of labor; and third, that the observed change in exports has had a positive net effect on job generation.
\end{abstract}

Keywords: Manufacturing exports, technological level, employment, structural decomposition analysis.

JEL Classification: F14, F16.

\section{Exportações manufatureiras mexicanas por nível tecnológico e seu efeito sobre o emprego em 2008 e 2012: uma análise de descomposição estrutural}

\begin{abstract}
Resumo
A composição das exportações manufatureiras por nível tecnológico usou-se para estimar, através da análise de descomposição estrutural e do valor da produção bruta do México de 2008 e 2012, as mudanças no emprego por unidade de variação nas exportações de cada subsetor. Os resultados fundamentais são três: primeiro, que são os subsetores manufatureiros de meia e alta tecnologia os que geram menos empregos por unidade de exportação; segundo, que são estes subsetores os que incrementaram em maior medida suas exportações de forma que seus requerimentos de emprego aumentaram mais que os do resto dos subsetores; e terceiro, que a mudança observada nas exportações tem tido um efeito positivo na criação de emprego.
\end{abstract}

Palavras-chave: exportações manufatureiras, níveis tecnológicos, emprego, análise de descomposição estrutural.

Classificação JEL: F14, F16. 


\section{Introducción}

Las exportaciones son uno de los determinantes importantes del crecimiento económico. A nivel macroeconómico, por una parte, debido a su papel de generador de las divisas que una economía requiere para producir y crecer; y, por otra, por el ingreso interno y el empleo que genera la producción de bienes destinados a la exportación.

Sin embargo, no solo es fundamental la magnitud del valor de las exportaciones. Diferentes estructuras de exportación tienen implicaciones distintas para el crecimiento y el desarrollo industrial de una economía. Este tema ha sido abordado desde diversas perspectivas. Una ha puesto énfasis en que en el mercado mundial el crecimiento de los volúmenes intercambiados difiere según el tipo de productos. Ya Prebisch (1949) había destacado que la demanda mundial por productos primarios crece más lentamente que la de productos manufacturados.

Una segunda perspectiva pone énfasis en que estructuras exportadoras de distintos niveles tecnológicos tienen efectos diferenciados sobre el resto de la economía. De acuerdo con Lall (2000), las exportaciones de productos de tecnología simple muestran mercados que crecen lentamente, menores posibilidades de mejoras tecnológicas y menor efecto de derrame sobre otras actividades. En cambio, las economías en las que predomina el uso de tecnologías avanzadas muestran senderos de crecimiento más elevados, a lo que contribuye el hecho de que la tecnología ofrece mayor potencial para la generación de conocimiento genérico. En este sentido, Lall et al. (2006) y Hausmann et al. (2007) han destacado que los países desarrollados se caracterizan por una estructura exportadora en la que predominan los productos de elevada intensidad tecnológica, mientras que en los países en desarrollo estos muestran bajo nivel de sofisticación y calidad en sus exportaciones.

El dinamismo de las manufacturas de alto y mediano nivel tecnológico ha llevado a que tengan una creciente participación en las exportaciones mundiales. En los últimos años se ha observado que algunos nuevos países industrializados han ido incrementando su participación tanto en las exportaciones mundiales como en las de productos de mayor intensidad tecnológica (UNCTAD, 2002). Estos países se han incorporado a la exportación intensiva en tecnología con productos cuyos procesos de producción están muy fragmentados y lo han hecho participando en las fases productivas intensivas en trabajo (Lall et al., 2006).

México ha sido uno de los países que ha modificado profundamente su estructura exportadora a favor de las manufacturas intensivas en tecnología. Según Fujii y García (2015), cerca del 50\% de las exportaciones mexicanas 
son de bienes intensivos en tecnología (datos para 1995 y 2011), en cuya producción México contribuye básicamente con el ensamblaje de productos mediante el uso de la ventaja que le confieren sus bajos salarios.

La liberalización comercial y el dinamismo de las exportaciones, junto con el cambio en la composición de las exportaciones, han tenido efectos importantes sobre el empleo. El objetivo de este trabajo es conocer el comportamiento de la cantidad de empleo generado por el cambio en las exportaciones manufactureras de México según niveles tecnológicos entre 2008 y 2012. La estimación se hace para los 21 subsectores que componen la industria manufacturera en México según el scian.

Si bien diversos autores han estudiado el efecto que la apertura comercial ha tenido sobre la generación de empleo en México, los trabajos que examinan el papel de la tecnología y de los cambios en la composición tecnológica de las exportaciones manufactureras sobre el empleo son escasos.

Las investigaciones recientes se han concentrado básicamente en los efectos de la liberalización comercial y del cambio en el perfil exportador del país sobre los niveles de empleo. Se ha destacado que mientras el efecto de estos procesos sobre el crecimiento de las exportaciones ha sido significativo, el impacto en el nivel de empleo no fue el esperado. Dussel-Peters (2004) argumenta que, a partir de la apertura comercial, los sectores de bienes transables generaron empleos muy por debajo de la contribución que hicieron durante las décadas anteriores a la apertura. Ruiz-Nápoles (2004) busca explicar el bajo efecto empleo de las exportaciones en el país, sosteniendo que ello se debe principalmente a la elevada importación de bienes intermedios, lo que hace que el empleo indirecto generado por las exportaciones sea muy bajo. Por su parte, Cervantes y Fujii (2012) encontraron que el efecto neto de la liberalización comercial sobre la generación de empleo ha sido positivo debido a la reestructuración del empleo a favor de los sectores manufactureros intensivos en trabajo no calificado. Aunque el contenido importado de las exportaciones en los sectores que aportan una parte significativa de las exportaciones manufactureras del país es muy elevado (79\% en las de la electrónica) y más reducido en las exportaciones de equipo de transporte (50\% de valor de sus exportaciones), el hecho de que México se haya especializado en las fases intensivas en trabajo del proceso de producción de estos productos determina que el número de puestos de trabajo directos e indirectos contenido en sus exportaciones sea importante: 989.7 y 459.9 mil, respectivamente (estos datos, para 2012, representan el $25,4 \%$ y el 11,8 \% del empleo total contenido en las exportaciones manufactureras del país). El elevado contenido importado de las exportaciones de la industria electrónica explica que el empleo 
contenido en ellas sea fundamentalmente directo (340 mil puestos de trabajo), mientras que el contenido en las exportaciones de equipo de transporte se divide entre 397 mil empleos directos y 592.8 mil empleos indirectos (ver Fujii et al., 2016).

La estructura del trabajo es la siguiente: en el apartado 1 se presenta la metodología utilizada tanto para clasificar los subsectores manufactureros por niveles tecnológicos como para cuantificar el efecto que el cambio en las exportaciones ha tenido sobre el empleo. En el 2 se presenta la composición de las exportaciones por niveles tecnológicos y en el 3 se expone el efecto del cambio en las exportaciones sobre el empleo manufacturero por niveles tecnológicos de las exportaciones manufactureras para los años 2008 y 2012. Finalmente, se presentan las conclusiones del trabajo.

\section{Metodología}

Las exportaciones manufactureras se clasifican por niveles tecnológicos de acuerdo con la clasificación realizada por Lall (2000). Esta clasificación es el resultado de la combinación de la metodología utilizada por Pavitt (1984) y la realizada por la OCDE (1994) para clasificar los productos por tecnología. La primera de ellas distingue entre manufacturas basadas en recursos, intensivas en trabajo, intensivas en escala, diferenciadas y basadas en ciencia; la segunda es una clasificación más detallada de la actividad tecnológica de cada categoría de Pavitt. El cambio en la composición de las exportaciones por niveles tecnológicos incide sobre el nivel de empleo por tres vías: i) al incrementarse el empleo por efecto de la cantidad de trabajo contenido en las exportaciones que están creciendo; ii) este efecto se amplifica o se aminora por cambios en el coeficiente de empleo por unidad de producción según los diferentes niveles tecnológicos; y iii) el cambio en la matriz de Leontief que refleja el cambio en la técnica de producción utilizada por el subsector en cuestión.

Para conocer el comportamiento de la cantidad de empleo generado por el cambio en las exportaciones manufactureras mexicanas según niveles tecnológicos entre 2008 y 2012, es necesario, primero, clasificar las exportaciones manufactureras por nivel tecnológico. Segundo, estimar la cantidad de empleo generado por el cambio en las exportaciones manufactureras entre 2008 y 2012 considerando sus tres causas directas: la modificación de los coeficientes de empleo, de la composición de las exportaciones y de los coeficientes técnicos de la matriz de Leontief. 


\subsection{Clasificación de las exportaciones por niveles tecnológicos}

En Lall (2000), se clasifican las exportaciones de $230^{1}$ grupos de productos de la Clasificación Uniforme del Comercio Internacional (CUCI a tres dígitos) de acuerdo con su nivel tecnológico en cinco niveles: productos primarios, manufacturas basadas en recursos naturales y manufacturas de baja, media y alta tecnología. Este trabajo solo considera los cuatro niveles tecnológicos referidos a las manufacturas (tabla 1$)^{2}$

Como las estadísticas disponibles para México sobre exportaciones y empleo que reporta el Instituto Nacional de Estadística y Geografía (Inegi) están clasificadas conforme con el ScIAN, fue necesario homologar los sistemas de clasificación industrial y del comercio. Hasta la fecha, no se ha elaborado la correspondencia directa entre las clasificaciones sCIAN y CUCI. Para elaborarla, nos hemos basado en los tabulados que muestran la equivalencia entre el SCIAN y la Clasificación Industrial Internacional Uniforme (CIIU) (UNSTATS, 2016), y entre la cIIU y la CUCI (CEPAL, 2008).

Tabla 1. Clasificación tecnológica de las exportaciones manufactureras

\begin{tabular}{lll}
\hline Código & & \multicolumn{1}{c}{ Descripción } \\
\hline (RB) & Manufacturas basadas en recursos naturales \\
& RB1 & Productos agroindustriales y de explotación forestal \\
& RB2 & Otros: manufacturas de la minería y la industria de la extracción \\
(LT) & Manufacturas de baja tecnología \\
& LT1 & Productos textiles, prendas de vestir y calzado \\
& LT2 & Otros: cerámica, muebles, juguetes, etc. \\
(MT) & Manufacturas de tecnología media \\
& MT1 & Productos automotrices \\
MT2 & Industria de procesos \\
MT3 & Industrias de ingeniería \\
Manufacturas de alta tecnología & Productos eléctricos y electrónicos \\
HT1 & Otros: industria farmacéutica, medicinal, aeroespacial, instrumentos \\
\hline
\end{tabular}

Fuente: elaboración de los autores con base en Lall (2000).

1 Se excluye cine, transacciones especiales, oro, obras de arte, monedas y mascotas.

2 La clasificación completa puede verse en Lall (2000). 
La homologación entre las tres clasificaciones (SCIAN, CIIU, CUCI) se realizó a nivel de rama, es decir, considerando la clasificación SCIAN a cuatro dígitos (ver anexo 1). Con la información del nivel tecnológico por ramas se agruparon las ramas en subsectores y estos se clasificaron por niveles tecnológicos. La principal limitante a la que nos enfrentamos fue el hecho de que un solo subsector scian puede contener productos de dos o más subcategorías diferentes de la clasificación por niveles tecnológicos de Lall. Para decidir a qué nivel tecnológico corresponden las exportaciones de estos subsectores, establecimos que su nivel tecnológico está definido por el nivel tecnológico de los productos exportados que contribuyen con, al menos, la mitad de las exportaciones de cada subsector.

\subsection{Análisis de descomposición estructural para la estimación del efecto} empleo del cambio en la composición tecnológica de las exportaciones

Para estimar la cantidad de empleo generado por el cambio en las exportaciones manufactureras entre 2008 y 2012, se realizó un análisis de descomposición estructural, que permite cuantificar el efecto que la variación en las exportaciones entre un año y otro tuvo sobre el nivel de empleo. El cálculo se basa en las matrices internas de insumo-producto de la economía mexicana para esos años, ambas a precios de 2008. El análisis se centró en los 21 subsectores de la industria manufacturera.

La idea central de este análisis es que los cambios de una variable dada se descompongan, de forma aditiva, en cambios en los factores determinantes de la variable (Schuschny, 2005). En este caso, se analizan los cambios en el nivel de empleo ocasionados por el cambio en el valor de las exportaciones. Considerando que la producción asociada a las exportaciones se determina a través de:

$$
x_{t}^{e}=L_{t} e_{t}
$$

donde $x_{t}^{e}$ es el vector de la producción asociada a las exportaciones $\left(e_{t}\right), L_{t}$ es la matriz inversa de Leontief $L_{t}=\left(I-A_{t}\right)^{-1}$, siendo $A$ la matriz de coeficientes técnicos de la matriz interna. El empleo generado por las exportaciones está determinado por el coeficiente de uso de empleo multiplicado por el nivel de producción asociado a dichas exportaciones:

$$
l_{t}^{e}=\hat{\lambda}_{t} x_{t}^{e}
$$

Sustituyendo la ecuación (1) en la (2) tenemos que el empleo asociado a las exportaciones también puede expresarse como: 


$$
l_{t}^{e}=\hat{\lambda}_{t} L_{t} e_{t}
$$

donde $l_{t}^{e}$ es el vector columna de empleos por rama generados por las exportaciones, $\hat{\lambda}_{\mathrm{t}}$ es el vector diagonalizado de los coeficientes de empleo que mide el número de empleos utilizados por unidad de producto y $L_{t} e_{t}$ es el vector columna de unidades producidas asociadas a las exportaciones y que se obtiene al multiplicar la matriz $L_{t}$ por el vector columna $e_{t}$.

Por lo tanto, el cambio en el empleo asociado a las exportaciones de una rama puede descomponerse, en forma aditiva, en el cambio de sus determinantes. Considerando el modelo de la ecuación (3) para dos períodos $t$ y $t-1$, se tiene que el cambio en el empleo se define como:

$$
l^{e}=l_{t}^{e}-l_{t-1}^{e}=\hat{\lambda}_{t} L_{t} e_{t}-\hat{\lambda}_{t-1} L_{t-1} e_{t-1}
$$

Sumando dos ceros $\left(\hat{\lambda}_{t-1} L_{t} e_{t}-\hat{\lambda}_{t-1} L_{t} e_{t}\right)$ y $\left(\hat{\lambda}_{t-1} L_{t-1} e_{t}-\hat{\lambda}_{t-1} L_{t-1} e_{t}\right)$ al lado derecho de la ecuación ( $\left.3^{\prime}\right)$, tenemos que el cambio en el empleo también se escribe como:

$$
\Delta l^{e}=l_{t}^{e}-l_{t-1}^{e}=\hat{\lambda}_{t} L_{t} e_{t}-\hat{\lambda}_{t-1} L_{t} e_{t}+\hat{\lambda}_{t-1} L_{t} e_{t}-\hat{\lambda}_{t-1} L_{t-1} e_{t}+\hat{\lambda}_{t-1} L_{t-1} e_{t}-\hat{\lambda}_{t-1} L_{t-1} e_{t-1}
$$

Agrupando los términos de acuerdo con los cambios en cada variable, se obtiene que el cambio en el empleo generado por las exportaciones se descompone como en la ecuación (4):

$$
\Delta l^{e}=l_{t}^{e}-l_{t-1}^{e}=\Delta \hat{\lambda} L_{t} e_{t}+\hat{\lambda}_{t-1} \Delta L e_{t-1}+\hat{\lambda}_{t-1} L_{t} \Delta e
$$

Análogamente, sumando dos ceros $\left(\hat{\lambda}_{t} L_{t-1} e_{t-1}-\hat{\lambda}_{t} L_{t-1} e_{t-1}\right)$ y $\left(\hat{\lambda}_{t} L_{t-1} e_{t}-\hat{\lambda}_{t} L_{t-1} e_{t}\right)$ al lado derecho de la ecuación ( $3^{\prime}$ ) y agrupando los términos, se obtiene que el cambio en el empleo también se puede descomponer como en la ecuación (5):

$$
\Delta l^{e}=l_{t}^{e}-l_{t-1}^{e}=\Delta \hat{\lambda} L_{t-1} e_{t-1}+\hat{\lambda}_{t} \Delta L e_{t}+\hat{\lambda}_{t} L_{t} \Delta e_{t}
$$

Las ecuaciones (4) y (5) son las descomposiciones polares-espejo ${ }^{3}$ del producto asociado a las exportaciones e indican que el cambio en el empleo

3 Son las descomposiciones que se obtienen de intercambiar entre sí el tiempo. La ecuación (4) pondera las variaciones en el coeficiente de empleo con la matriz de Leontief 
generado por las exportaciones $\left(\Delta_{l}^{e}\right)$ es el resultado de tres efectos: el cambio en el coeficiente de empleo $(\Delta \hat{\lambda})$, el cambio en la matriz de Leontief $(\Delta L)$ y el cambio en el valor de las exportaciones $(\Delta e)$, ponderados cada uno por $\hat{\lambda}, L$ y $e$ en los diferentes períodos $t$ y $t-1$.

El número de descomposiciones posibles crece con la cantidad de variables que participan en ella. ${ }^{4}$ No obstante, en diversos trabajos se ha demostrado que el promedio de todas las descomposiciones posibles se aproxima al promedio de las dos descomposiciones polares-espejo que se obtienen de intercambiar el tiempo en las variables que participan en la descomposición (Dietzenbacher y Los, 1998; De Haan, 2001).

Finalmente, el promedio de las dos descomposiciones polares-espejo nos permitirá obtener la descomposición del cambio en el empleo asociado a las exportaciones generado por sus determinantes (ver tabla 2).

Tabla 2. Descomposición del empleo asociado a las exportaciones

$$
\begin{aligned}
& \Delta l^{e}=\quad \frac{1}{2}\left(\Delta \hat{\lambda} L_{t} e_{t}+\Delta \hat{\lambda} L_{t-1} e_{t-1}\right) \\
& +\frac{1}{2}\left(\hat{\lambda}_{t-1} \Delta L e_{t-1}+\hat{\lambda}_{t} \Delta L e_{t}\right) \quad \text { Matriz de Leontief } \\
& +\frac{1}{2}\left(\hat{\lambda}_{t-1} L_{t} \Delta e+\hat{\lambda}_{t} L_{t-1} \Delta e\right) \quad \text { Exportaciones }
\end{aligned}
$$

Fuente: elaboración de los autores.

El primer término de la tabla 2 denota el cambio en el empleo asociado a las exportaciones ocasionado por el cambio en el coeficiente de empleo, es decir, por el cambio en la intensidad de uso del factor trabajo. El segundo término indica el cambio en el empleo generado por el cambio en la matriz de Leontief, es decir, en la estructura de producción y distribución de los diferentes sectores. El último término permite identificar el empleo generado o desplazado en un determinado período a causa del incremento o disminución en las exportaciones; este último es el de mayor relevancia en este trabajo, ya que vincula el cambio en el empleo con el cambio observado en las exportaciones; por ello, renombramos a este último término como en la ecuación (6):

y las exportaciones del período $t$, mientras que la ecuación (5) las pondera con la matriz de Leontief y las exportaciones en $t-1$.

4 El número de descomposiciones posible es $n$ !, donde $n$ es el número de variables determinantes del modelo (ver Dietzenbacher y Los, 1998). 


$$
\Delta l^{e s e}=\frac{1}{2}\left(\hat{\lambda}_{t-1} L_{t} \Delta e+\hat{\lambda}_{t} L_{t-1} \Delta e\right)
$$

Premultiplicando ambos lados de la ecuación (6) por la matriz inversa diagonalizada del cambio en las exportaciones $(\Delta e)$, se obtiene un multiplicador del empleo que mide el cambio en el empleo por unidad de variación en la demanda de exportación:

$$
\widehat{\Delta e}^{-1} \Delta l^{e \Delta e}=\widehat{\Delta e}^{-1}\left[\frac{1}{2}\left(\hat{\lambda}_{t-1} L_{t} \Delta e+\hat{\lambda}_{t} L_{t-1} \Delta e\right)\right]
$$

La ecuación (7) indica que el empleo generado en cada sector por la modificación en sus exportaciones resulta de la suma de dos efectos: el primero capta el empleo generado en cada sector por unidad producida a causa del cambio en sus exportaciones; y el segundo representa el empleo generado en cada sector por unidad producida a causa del cambio en las exportaciones de los otros sectores. En otras palabras, la ecuación (7) representa los efectos directos e indirectos que el cambio en las exportaciones tiene sobre el empleo.

\section{Composición de las exportaciones mexicanas por nivel tecnológico}

En la tabla 3 se muestran los subsectores manufactureros clasificados según su nivel tecnológico. En la tercera y quinta columnas se muestran las ramas SCIAN que, en 2008 y 2012, contribuyeron con más del $50 \%$ de las exportaciones de dicho subsector. $\mathrm{Y}$ las columnas cuatro y seis muestran el nivel tecnológico de las exportaciones que predomina en las ramas que conforman el subsector en cuestión.

Es evidente que las exportaciones de las industrias de alimentos, bebidas y tabaco, madera, petróleo y carbón se caracterizan por ser manufacturas basadas en recursos naturales (RB). Por su parte, aquellas exportaciones de las industrias relacionadas con los textiles, prendas de vestir, piel, cuero, minerales no metálicos, muebles, colchones y otras manufacturas son manufacturas de baja tecnología (LT). Además, las exportaciones de las industrias de los productos metálicos, maquinaria y equipo, cómputo, aparatos eléctricos, transporte, plástico y hule representan manufacturas de tecnología media y alta (мт, нт). Resaltan tres casos en los que la clasificación experimentó cambios entre 2008 y 2012. 
Tabla 3. Subsectores manufactureros por niveles tecnológicos de acuerdo con sus exportaciones, 2008 y 2012

\begin{tabular}{|c|c|c|c|c|c|c|c|c|c|}
\hline \multirow{2}{*}{$\begin{array}{l}\text { Sub- } \\
\text { sector } \\
\text { SCIAN }\end{array}$} & \multirow[b]{2}{*}{ Descripción } & \multicolumn{4}{|c|}{2008} & \multicolumn{4}{|c|}{2012} \\
\hline & & & Ramas & & $\begin{array}{l}\text { Nivel tecno- } \\
\text { lógico }\end{array}$ & & Ramas & & $\begin{array}{l}\text { Nivel tecnoló- } \\
\text { gico }\end{array}$ \\
\hline 311 & Ind. alimentaria & 3113 & 3114 & 3119 & RB1 & 3113 & 3114 & 3116 & RB1 \\
\hline 312 & Bebidas y tabaco & 3121 & & & RB1 & 3121 & & & RB1 \\
\hline 313 & Insumos textiles & 3131 & 3133 & 3132 & LT1 & 3132 & 3131 & & LT1 \\
\hline 314 & Productos textiles & 3141 & 3149 & & LT1 & 3149 & & & LT1 \\
\hline 315 & Prendas de vestir & 3152 & & & LT1 & 3152 & & & LT1 \\
\hline 316 & Ind. piel y cuero & 3169 & 3162 & 3161 & LT1 & 3162 & 3161 & & LT1 \\
\hline 321 & Ind. madera & 3219 & & & RB1 & 3219 & & & RB1 \\
\hline 322 & Ind. papel & 3222 & & & RB1, LT1, LT2 & 3222 & & & RB1, LT1, LT2 \\
\hline 323 & Impresión y conexas & 3231 & & & LT2, MT2 & 3231 & & & LT2, MT2 \\
\hline 324 & $\begin{array}{l}\text { Deriv. petróleo y } \\
\text { carbón }\end{array}$ & 3241 & & & RB2 & 3241 & & & RB2 \\
\hline 325 & Ind. química & 3251 & 3252 & 3254 & RB2, MT2 & 3251 & 3252 & 3256 & MT2, RB2 \\
\hline 326 & Ind. plástico y hule & 3261 & & & MT2 & 3261 & & & MT2 \\
\hline 327 & $\begin{array}{l}\text { Ind. minerales no } \\
\text { metálicos }\end{array}$ & 3271 & 3272 & & LT1 & 3271 & 3272 & & LT1 \\
\hline 331 & $\begin{array}{l}\text { Ind. metálicas bá- } \\
\text { sicas }\end{array}$ & 3314 & 3311 & & $\mathrm{PP}^{*}, \mathrm{MT} 2$ & 3314 & & & $\mathrm{PP}^{*}$ \\
\hline 332 & $\begin{array}{l}\text { Productos metá- } \\
\text { licos }\end{array}$ & 3329 & 3325 & & HT1, MT3 & 3329 & 3325 & & HT1, MT3 \\
\hline 333 & Maquinaria y equipo & 3336 & 3334 & 3339 & HT1, MT3 & 3336 & 3334 & 3339 & HT1, MT3 \\
\hline 334 & $\begin{array}{l}\text { Cómputo, comu- } \\
\text { nicac. }\end{array}$ & 3344 & 3343 & & HT1, MT3 & 3343 & 3341 & 3342 & HT1, MT3 \\
\hline 335 & $\begin{array}{l}\text { Acces. y aparatos } \\
\text { eléctricos }\end{array}$ & 3353 & 3359 & & HT1, MT3 & 3353 & 3359 & & HT1, MT3 \\
\hline 336 & $\begin{array}{l}\text { Equipo de trans- } \\
\text { porte }\end{array}$ & 3363 & 3361 & & MT3, MT1 & 3361 & 3363 & & MT1, MT3 \\
\hline 337 & $\begin{array}{l}\text { Muebles, colchones, } \\
\text { etc. }\end{array}$ & 3371 & & & LT2 & 3371 & & & LT2 \\
\hline 339 & Otras manufacturas & 3391 & & & LT2 & 3391 & & & LT2 \\
\hline
\end{tabular}

Nota: la clasificación por niveles tecnológicos de Lall (2000) se basa en la cucI; en esta, la definición de manufacturas (categorías de la 5 a la 8 menos la 68: metales no ferrosos) es más estricta que aquella usada por la ciru (ver Wood y Mayer, 2001). Por eso, de acuerdo con Lall, los productos de la rama 3314 (metales no ferrosos) se clasifican como productos primarios (PP).

Fuente: elaboración de los autores. 
El primero es el de la industria química (325), en la que en 2008 dominaban las exportaciones de manufacturas basadas en recursos naturales (RB2), mientras que en 2012 dominan las de tecnología media (MT2). En el caso del subsector de las industrias metálicas básicas (331), el nivel tecnológico de las exportaciones empeoró. Para 2012, el 69 \% de sus exportaciones totales fue de productos primarios o no manufactureros. El tercer caso es el del equipo de transporte (336); en 2008 se exportaron más productos de tecnología media relacionados con la ingeniería (MT3) que en 2012, año en que se exportaron más manufacturas automotrices (MT1).

Es importante hacer notar que en ninguno de los subsectores manufactureros predominan las exportaciones de alta tecnología de la subcategoría HT2, en la que se encuentran productos de las industrias farmacéutica, medicinal, aeroespacial, turbinas, instrumentos ópticos, entre otros.

En la tabla 4 se presenta la participación de cada uno de los niveles tecnológicos en el valor de las exportaciones. Se decidió crear la categoría HT1-MT3, ya que estas categorías siempre se presentan de manera conjunta.

Tabla 4. Exportaciones manufactureras mexicanas por nivel tecnológico, 2008 y 2012

(Participación porcentual y tasa media anual de crecimiento)

\begin{tabular}{lccc}
\hline \multicolumn{1}{c}{ Nivel tecnológico } & $\begin{array}{c}2008 \\
(\%)\end{array}$ & $\begin{array}{c}2012 \\
(\%)\end{array}$ & $\begin{array}{c}\text { Tasa media anual de } \\
\text { crecimiento }(\%)\end{array}$ \\
\hline $\begin{array}{l}\text { Total de las exportaciones manufacture- } \\
\text { ras (millones de pesos a precios de 2008) }\end{array}$ & $\begin{array}{c}100 \% \\
(2379596)\end{array}$ & $\begin{array}{c}100 \% \\
(3527184)\end{array}$ & 10,3 \\
PP & 5,0 & 5,5 & 13,0 \\
RB & 12,0 & 6,0 & $-7,2$ \\
LT & 10,0 & 7,5 & 2,7 \\
MT & 27,0 & 35,0 & 17,7 \\
HT1, MT3 & 46,0 & 46,0 & 10,3 \\
\hline
\end{tabular}

Nota: en 2008 y 2012, el nivel tecnológico PP contiene al sector 331; LT se compone por: 313, 314, 315, 316, 323, 327, 337 y 339; HT1, MT3 por: 332, 333, 334 y 335. En 2008, RB se compone por: 311, 312, 321, 322, 324 y 325; en 2012, el subsector 325 deja de formar parte de Rв y se incluye en мт. En 2008, мт se conforma por: 326 у 336; en 2012, también incluye al sector 325 .

Fuente: elaboración de los autores.

En términos generales, se observa un cambio en la estructura de las exportaciones manufactureras mexicanas en favor de productos con nivel tecnológico medio (MT) y medio-alto (HT1, MT3), y en detrimento de las exportaciones de nivel tecnológico bajo (LT) y basadas en recursos naturales (RB). En 2012, 
las exportaciones de nivel tecnológico medio y medio-alto representaron el $81 \%$ de las exportaciones manufactureras.

El nivel tecnológico que concentra la mayor parte del valor total de las exportaciones es el compuesto por los estratos de media y alta tecnología (MT3 y HT1); en ambos años representó el $46 \%$ del total de las exportaciones manufactureras. Por su parte, las exportaciones de tecnología media (MT1 y MT2) cobraron importancia entre 2008 y 2012; una de las razones de este cambio está en el que experimentó el subsector de la industria química (325), dado que sus exportaciones mostraron un cambio en su composición a favor de las exportaciones de tecnología media.

Por último, las exportaciones manufactureras que mostraron la tasa media de crecimiento anual más alta entre 2008 y 2012 fueron las de tecnología media (мт), con el 17,7\%, lo que contrasta con el comportamiento de las manufacturas basadas en recursos naturales (RB), cuyas exportaciones mostraron un decrecimiento medio anual del 7,2\%. Esto ocasionó que la estructura tecnológica de las exportaciones manufactureras transitara hacia manufacturas más intensivas en tecnología.

\section{Nivel tecnológico y el efecto del cambio en las exportaciones sobre el empleo}

La tabla 5 muestra el número de empleos generados por unidad de variación de las exportaciones, resultado del análisis de descomposición estructural (ver ecuación 7). El cálculo se hizo a nivel sectorial y los resultados se ordenaron de mayor a menor según el número de empleos creados por un millón de pesos de incremento en las exportaciones. Los datos fueron agrupados en cinco grupos, los cuales se analizan en detalle más adelante (ver tabla 6). En promedio, en los subsectores manufactureros del grupo 1, entre 2008 y 2012, un millón de pesos adicional de demanda de exportaciones generó 7,4 empleos adicionales. En contraste, aquellos subsectores que se encuentran en el grupo 4 generaron, entre los mismos años, 0,53 empleos adicionales por millón de pesos de incremento de la demanda de exportaciones. En los primeros cuatro grupos se encuentran 19 de los 21 subsectores. El primero está conformado por cuatro subsectores y los otros tres, por cinco subsectores cada uno. El grupo cinco se conforma por los dos subsectores que registraron información atípica. 
Tabla 5. Empleos generados por millón de pesos adicional de exportación, 2008 y 2012

\begin{tabular}{cccc}
\hline & Mínimo & Máximo & Media \\
\hline Grupo 1 & 3,62 & 11,66 & 7,38 \\
Grupo 2 & 2,02 & 2,69 & 2,37 \\
Grupo 3 & 0,71 & 1,84 & 1,30 \\
Grupo 4 & 0,43 & 0,71 & 0,53 \\
Grupo 5 & - & - & - \\
\hline
\end{tabular}

Fuente: elaboración de los autores con base en las matrices internas de 2008 y 2012 (Inegi, 2016).

En la tabla 6 se ilustra, a nivel de grupos y sectores, el cambio en el empleo por niveles tecnológicos derivado de la dinámica exportadora y el peso de los tres factores que hemos señalado (variaciones de las exportaciones, del coeficiente de empleo por unidad exportada y de la estructura productiva representada mediante la inversa de Leontief) en el comportamiento del empleo. Adicionalmente, la tabla señala los niveles tecnológicos de las exportaciones de cada sector, los coeficientes de empleo y de empleo por unidad de exportación por sector, y la variación en el valor de las exportaciones de cada sector entre 2008 y 2012. Las conclusiones más relevantes de esta información son las siguientes:

i) Entre los años considerados, el cambio en las exportaciones manufactureras en cuanto a valor y a composición derivó en la generación de un total de 311 mil empleos (columna 10), esta magnitud pareciera ser pequeña comparada con el tamaño de la economía mexicana. Sin embargo, entre 2008 y 2012, el empleo manufacturero mostró una variación de -220 mil empleos. Esto quiere decir que en el sector manufacturero las exportaciones han creado más empleos de los que en total se destruyen. No obstante, distintos factores, como el cambio técnico, la contracción del mercado interno, han reducido la capacidad de generación de empleo de manufactura mexicana.

ii) Este incremento se derivó del efecto positivo provocado por el aumento de las exportaciones, el que más que compensó el efecto negativo sobre el empleo derivado del descenso en el coeficiente de empleo de las exportaciones. Las exportaciones se incrementaron en 1.147.6 mil millones de pesos (columna 6), lo que generó 837 mil empleos (columna 7), mientras que el efecto del descenso del coeficiente de empleo tuvo un efecto 'destructor' de empleo que ascendió a 525 mil puestos de trabajo (columna 8). El efecto empleo asociado a los cambios de la matriz de Leontief fue muy pequeño (columna 9). 


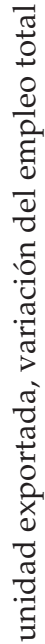

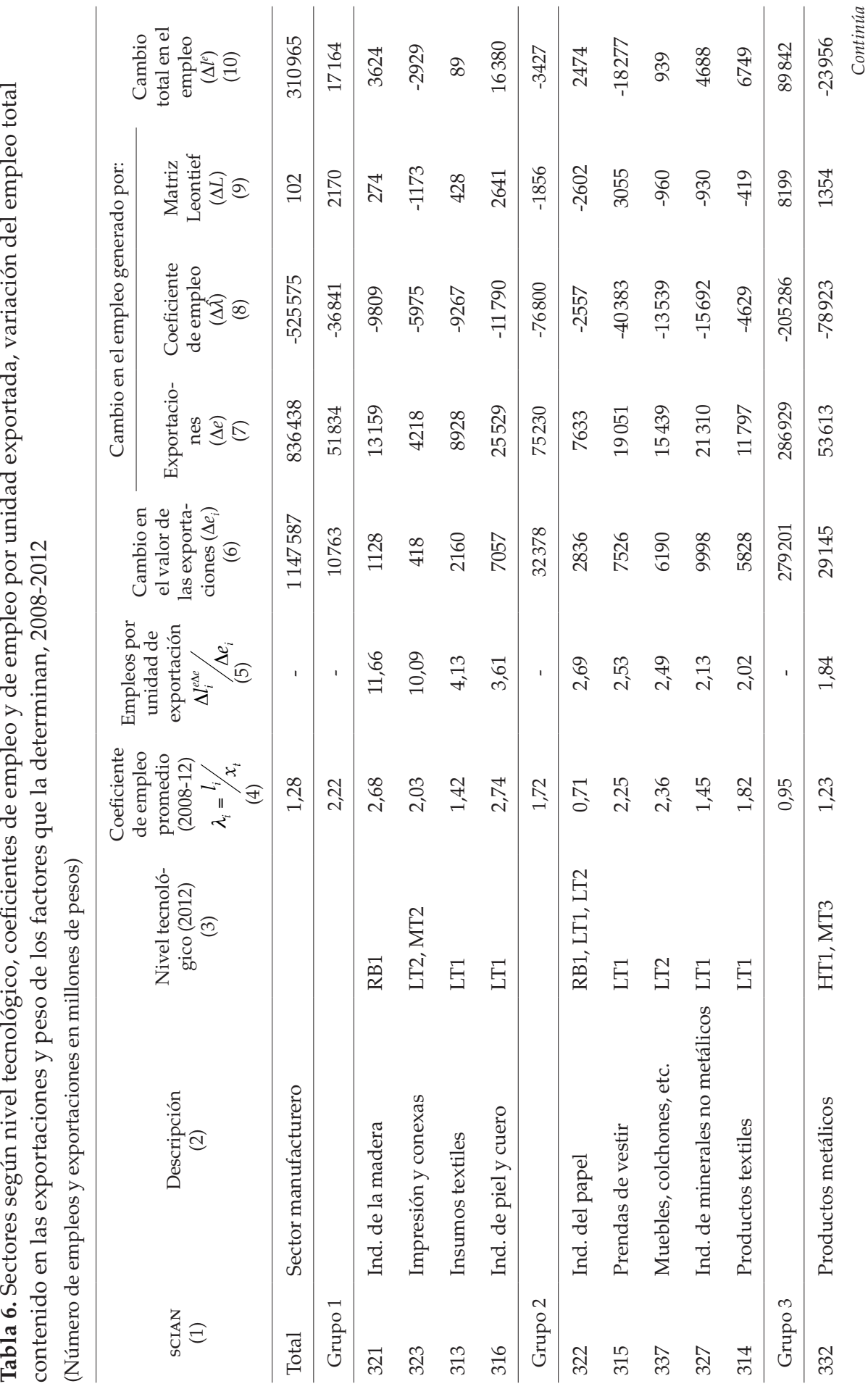




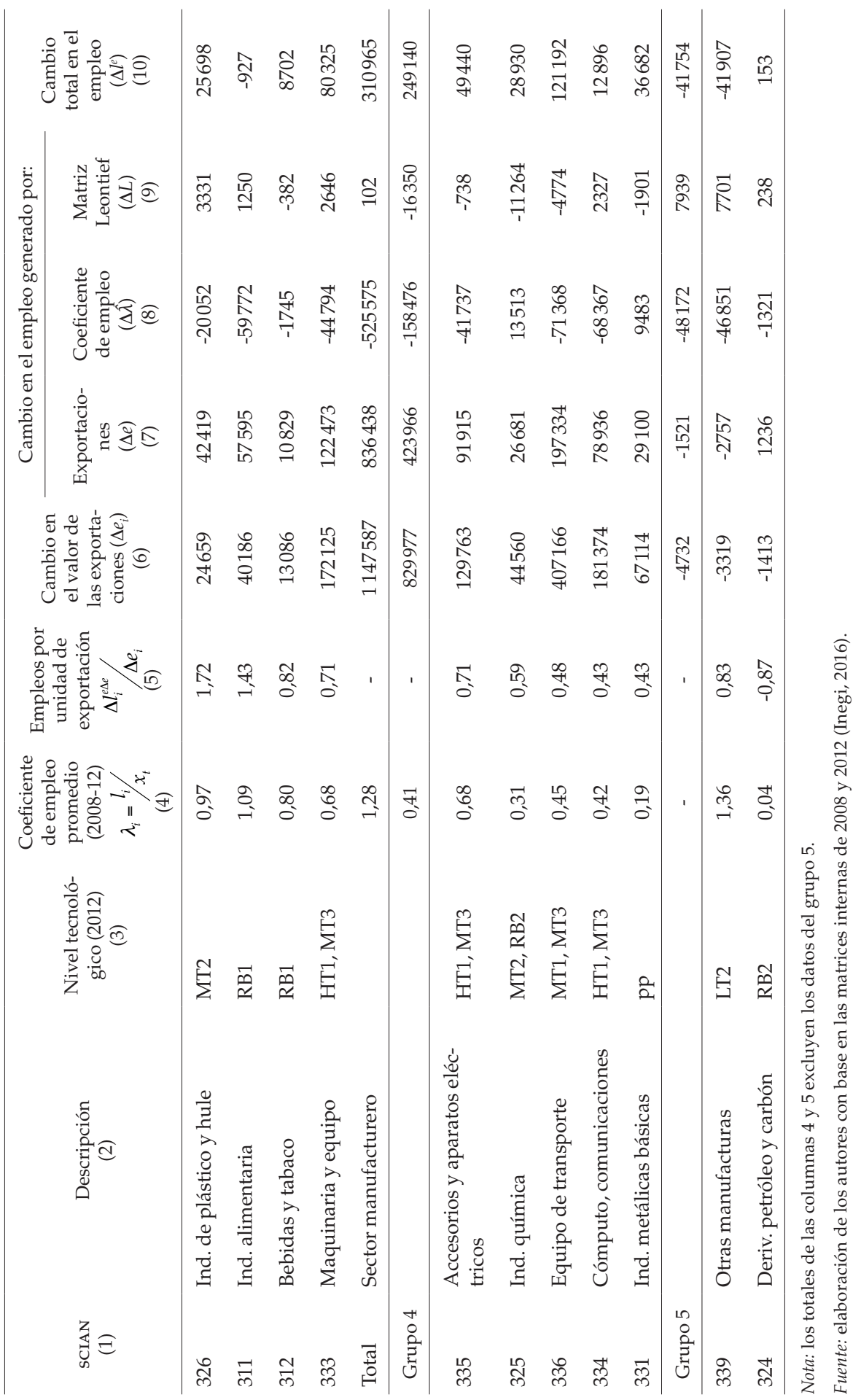


iii) Los mayores incrementos en el empleo se dieron en los grupos 3 y 4 . En ambos el sustancial incremento en las exportaciones compensó la 'destrucción' de empleos ocasionada por el descenso en el coeficiente de empleo, el primer grupo creó 90 mil empleos y el segundo 249 mil.

iv) Por su nivel tecnológico, en los grupos 3 y 4 dominan las manufacturas de tecnología media y alta. Los sectores que incluye el grupo 3 tienen coeficientes de empleo cercanos a la media total, mientras que el coeficiente de empleo por unidad adicional de exportación está por debajo de la media. Los subsectores que incluye el grupo 4 tienen una capacidad de generación de empleos baja tanto por sus reducidos coeficientes de empleo como de empleo marginal por unidad incremental de exportación. En este grupo se encuentran subsectores con niveles tecnológicos medio $\mathrm{y}$ alto integrados a las cadenas globales de valor y que menos empleos por unidad de producto generan. Estos son los casos de las industrias de fabricación de accesorios y aparatos eléctricos (335), de equipo de transporte (336) y de equipo de cómputo y comunicación (334). Una característica en común de los subsectores 334 y 335 es que, aunque sus productos son clasificados como de alta tecnología, la mayoría de las empresas de este rubro localizadas en México se especializan en la etapa final de la producción, es decir, en la etapa en la que se hace uso intensivo de mano de obra. Los requerimientos de habilidades en estas industrias tienden a ser entre simples e intermedias. Por su parte, la mano de obra empleada en el subsector 336 se caracteriza por estar más calificada en la medida que requiere habilidades avanzadas. Por lo tanto, aunque en las industrias integradas en cadenas globales de valor México se ha especializado en las fases intensivas en trabajo, el coeficiente de empleo de ellas es relativamente bajo, por lo que la generación de nuevos empleos depende fundamentalmente de su dinámica exportadora.

v) A nivel de subsectores, los que más empleo derivado de las exportaciones generaron fueron, en orden descendente, fabricación de equipo de transporte (336), maquinaria y equipo (333), accesorios y aparatos eléctricos (335) e industrias metálicas básicas (331). El segundo pertenece al grupo 3 y los restantes al 4. Las exportaciones de equipo de transporte (336) generaron 121 mil nuevos empleos (29\% del total), que resulta del efecto positivo por valor de las exportaciones, igual a 197 mil puestos de trabajo, a los que se les deduce el efecto negativo de 71 mil empleos por el descenso en el coeficiente de empleo. En el de maquinaria y equipo (333), las exportaciones generaron 80 mil empleos netos, que resultan de la creación de 122 mil puestos de trabajo por el comportamiento del valor de las exportaciones y la 'destrucción' de 45 mil empleos por el 
efecto del coeficiente de empleo. En el sector de accesorios y aparatos eléctricos (335) los datos respectivos son iguales a 49, 92 y 42 mil puestos de trabajo, y en la industria metálica básica (331), de 37, 29 y 10 mil empleos. El cambio en la estructura productiva 'destruyó' empleos en los subsectores 333, 335 y 336, y solo favoreció al subsector 331.

vi) Si ordenamos a los subsectores por el crecimiento absoluto de las exportaciones, tres (336, 335 y 333) están entre los que tuvieron los mayores incrementos en el empleo derivado de las exportaciones. En el subsector fabricación de equipo de cómputo y comunicación (334), que ocupa la segunda posición por el aumento en las exportaciones (181 mil millones de pesos), el incremento neto en la generación de empleo fue de solo 13 mil puestos de trabajo, lo que se explica porque fue el sector que experimentó el segundo mayor efecto negativo sobre el empleo por el descenso en el coeficiente de empleo (68 mil empleos), mientras que el efecto positivo derivado del comportamiento del valor de las exportaciones fue de 79 mil empleos.

vii) Los subsectores en los que el efecto exportaciones sobre el empleo fue negativo en una magnitud importante fueron el de otras manufacturas (339), productos metálicos (332) y prendas de vestir (315). En todos los casos este resultado se explica por la caída en el coeficiente de empleo. El primero registró un descenso de 42 mil puestos de trabajo, el descenso en el coeficiente de empleo 'destruyó' 48 mil empleos, a lo que se añadió el descenso en las exportaciones. En el subsector productos metálicos (332), el empleo derivado de las exportaciones cayó en 24 mil puestos de trabajo, lo que también es resultado del descenso en el coeficiente de empleo, que redujo los puestos de trabajo en 79 mil empleos, y que no fueron compensados con los creados por el incremento en las exportaciones. Por último, en el sector prendas de vestir (315), el empleo resultante de las exportaciones cayó en 18 mil puestos de trabajo, lo que resulta del efecto negativo determinado por el coeficiente de empleo (40 mil empleos), compensado parcialmente por el efecto positivo derivado del volumen de exportaciones (19 mil empleos).

viii) En síntesis, los mayores efectos generadores de empleo derivados de las exportaciones están en los subsectores de los grupos 3 y 4 , con tecnologías medias, con bajos coeficientes de empleo y de empleo por unidad exportada, por lo que es el crecimiento del valor de las exportaciones lo que explica la generación de empleo. Por otra parte, los sectores más intensivos en trabajo (grupos 1 y 2) fueron los menos dinámicos en la generación de empleo exportador, pues al efecto negativo derivado del comportamiento del coeficiente de empleo se le aunó una débil dinámica exportadora. 


\section{Conclusiones}

En las últimas décadas, las exportaciones mexicanas se han caracterizado por un elevado crecimiento y por un profundo cambio en su composición en favor de las manufacturas de niveles tecnológicos medios y altos, lo que ha afectado al empleo generado por las exportaciones.

El comportamiento del empleo generado por las exportaciones se ha descompuesto en tres determinantes directos: el comportamiento del valor de las exportaciones, la variación del coeficiente de empleo contenido en las exportaciones y el cambio de los coeficientes de la matriz inversa de Leontief.

La mayor parte del empleo creado por las exportaciones manufactureras entre 2008 y 2012 ha ocurrido en los sectores de niveles tecnológicos medio y alto, que tienen los menores coeficientes de empleo. Esto llama la atención por el hecho de que en una economía con excedentes de trabajo se esperaría que fueran los sectores intensivos en trabajo, con coeficientes de empleo elevados, los que deberían mostrar un dinamismo exportador significativo.

Parte de la respuesta a esta aparente paradoja radica en la elevada intensidad laboral de la fase del proceso productivo de los productos de tecnología media y alta producidos y exportados por México.

La creación de empleo en estos sectores se explica en gran medida por el crecimiento de las exportaciones, que más que compensó el efecto negativo del comportamiento del coeficiente de empleo por unidad exportada. El efecto de los cambios de los coeficientes de la matriz de Leontief sobre el empleo contenido en las exportaciones es menor.

El crecimiento más rápido de las exportaciones manufactureras intensivas en recursos naturales y de bajo nivel tecnológico, al tener coeficientes de empleo más elevados, podría tener un elevado efecto de creación de empleo tanto directo, en la fase manufacturera de la producción, como indirecto, por el eslabonamiento con los sectores primarios productores de las materias primas.

\section{Referencias}

CEPAL. (2008). Clasificaciones estadísticas internacionales incorporadas en el Banco de Datos del Comercio Exterior de América Latina y el Caribe de la CEPAL (revisión 3). Cuadernos Estadísticos, (3).

Cervantes, R., \& Fujii, G. (2012). The Mexican trade liberalization process and its net effects on employment: 1988-2004. EconoQuantum, 9(2), 81-97.

De Haan, M. (2001). A structural decomposition analysis of pollution in the Netherlands. Economic Systems Research, 13, 181-196. 
Dietzenbacher, E., \& Los, B. (1998). Structural decomposition techniques: sense and sensitivity. Economic Systems Research, 10, 307-323.

Dussel-Peters, E. (2004). Efectos de la apertura comercial en el empleo y en el mercado laboral de México y sus diferencias con Argentina y Brasil (1990-2003). Documentos de Estrategias de Empleo, ort.

Fujii, G., \& García, M. (2015). Revisiting the quality of exports. Journal of Economic Structures, 4(17).

Fujii, G., Cervantes, R., \& Fabián, A. S. (2016). Contenido de trabajo en las exportaciones manufactureras mexicanas, 2008 y 2012. Revista CEPAL, 119, 167-186.

Hausmann, R., Hwang, J., \& Rodrik, D. (2007). What you export matters. Journal of Economic Growth, 12(1), 1-25.

Inegi. (2016). Matrices de insumo-producto. Recuperado de http:/ / www.inegi. org.mx/

Lall, S. (2000). The technological structure and performance of developing country manufactures exports, 1985-1998. Oxford Development Studies, 28, 337-369.

Lall, S., Weiss, J., \& Zhang, J. (2006). The sophistication of exports: a new trade measure. World Development, 34(2), 222-237.

Pavitt, K. (1984). Sectoral patterns of technical change: towards a taxonomy and a theory. Research Policy, 13, 343-373.

Prebisch, R. (1949). El desarrollo económico de América Latina y algunos de sus principales problemas. E/CN.12/89, Santiago de Chile.

Ruiz-Nápoles, P. (2004). Exports, growth and employment in México, 19782000. Journal of Post Keynesian Economics, 27(1), 105-124.

Schuschny, A. (2005). Tópicos sobre el modelo de insumo-producto. Teoría y aplicaciones. Serie de Estudios Estadísticos y Prospectivos. Santiago: CEPAL, División de Estadística y Proyecciones Económicas.

unCTAD. (2002). Trade and development report. Geneva: United Nations Conference on Trade and Development.

unSTATs. (2016). Correspondence between NAICS and IIIC. United Nations Statistics Division. Recuperado de http:/ / unstats.un.org/

Wood, A., \& Mayer, J. (2001). Africa's export structure in a comparative perspective. Cambridge Journal of Economics, 25(3), 369-394. 


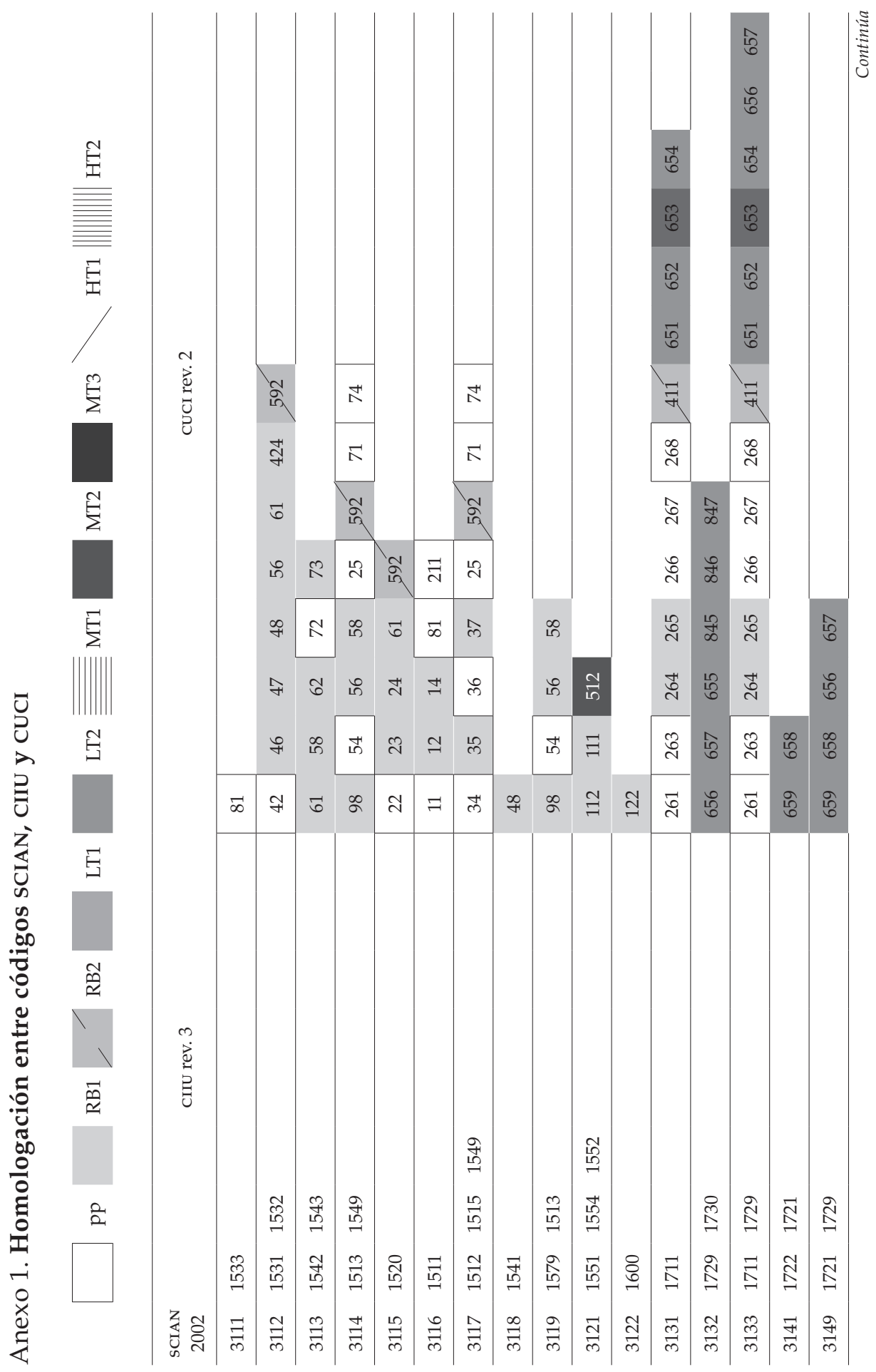




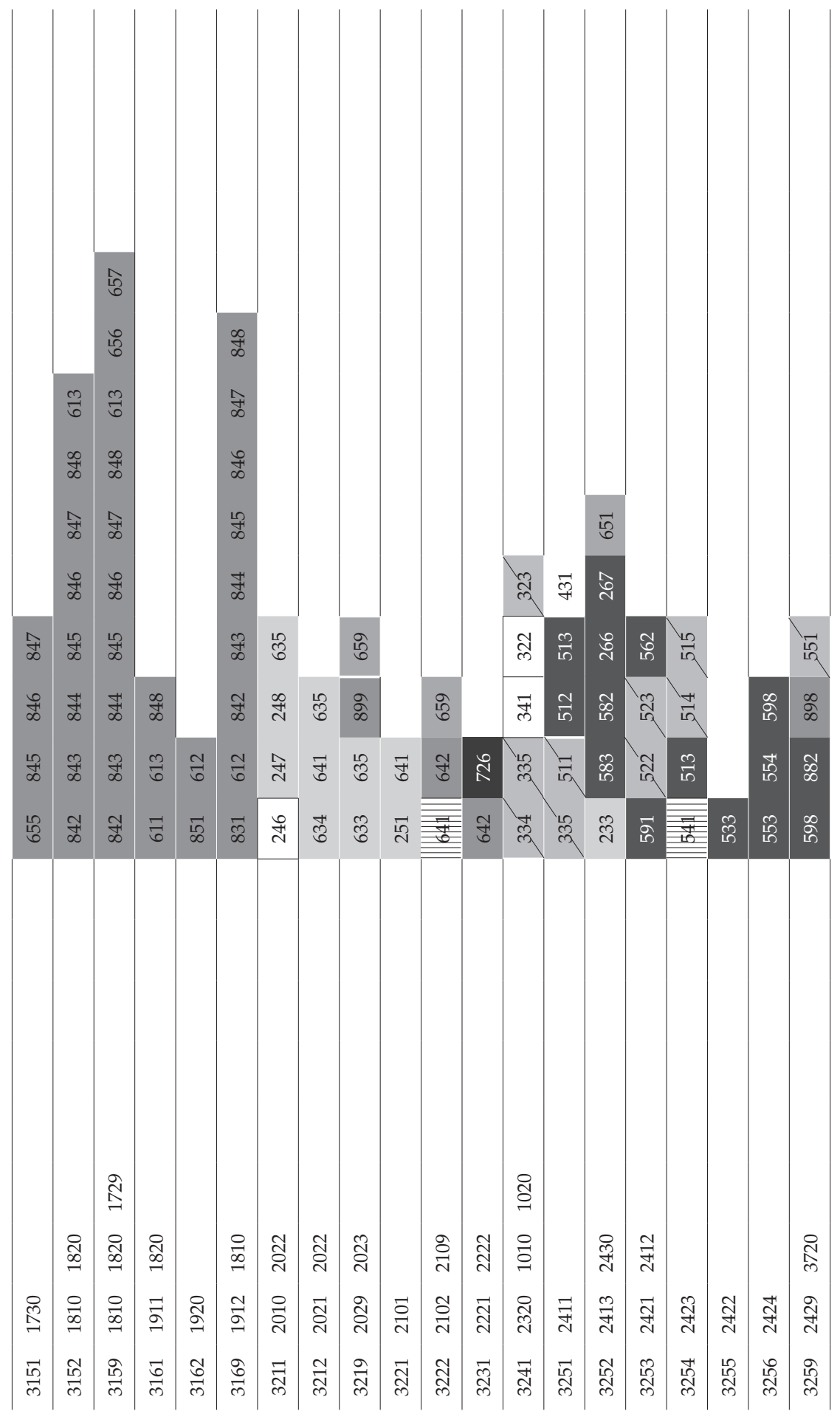









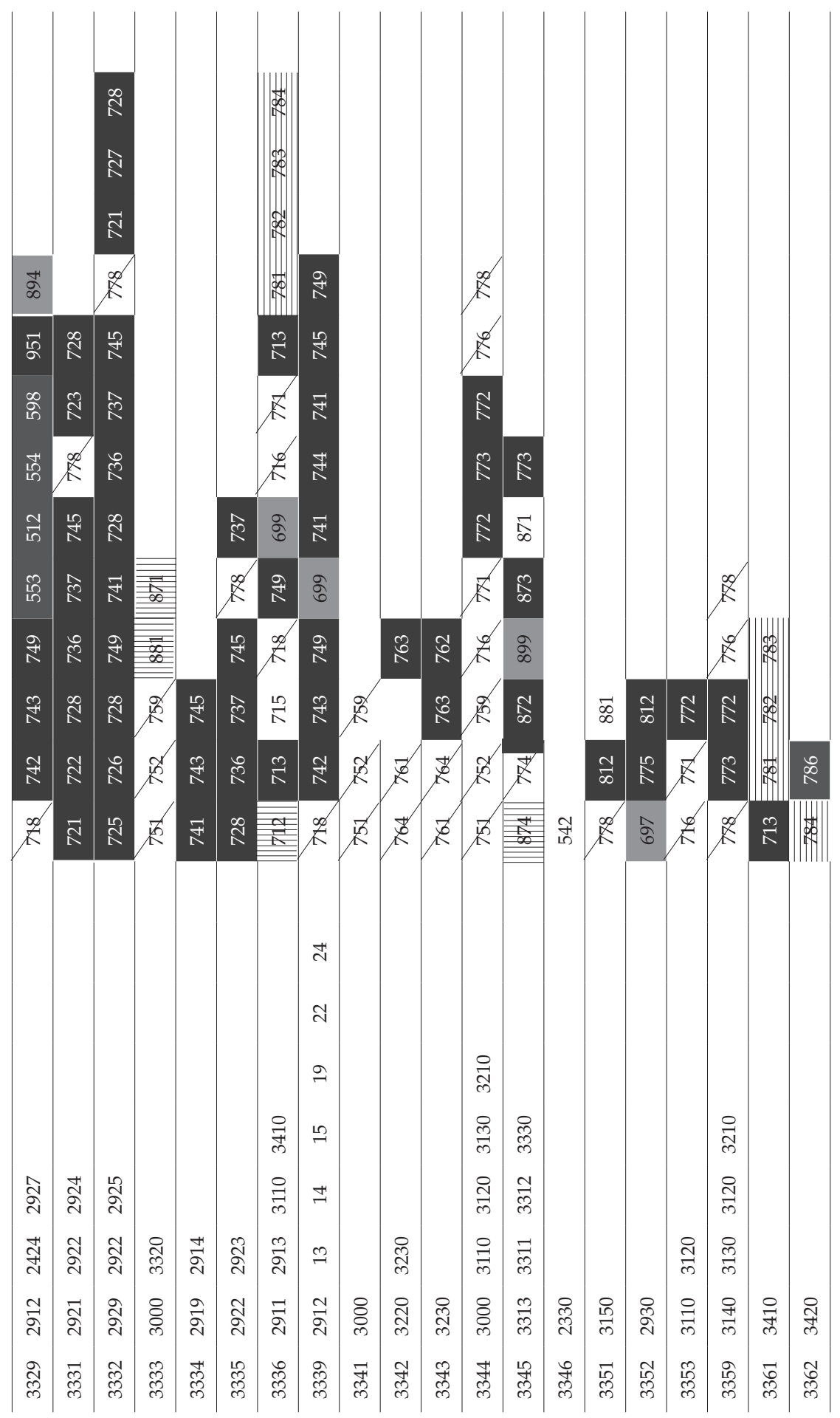




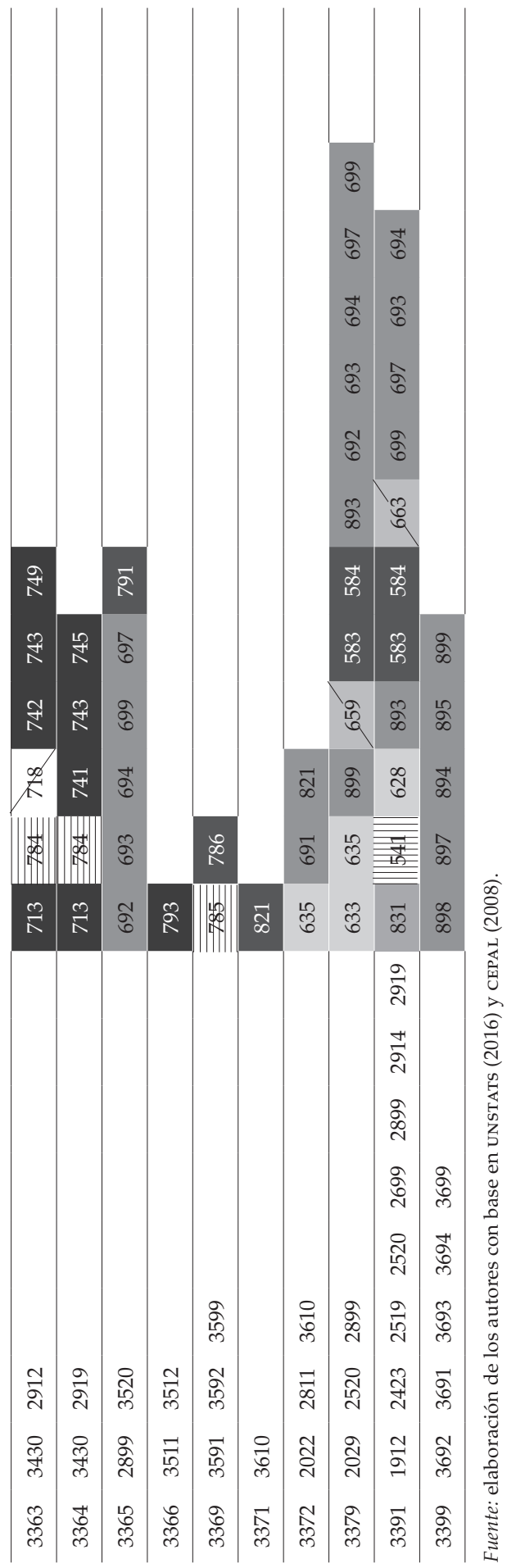


